\title{
Producción de abono orgánico mediante el compostaje aerotérmico de residuos de poda
}

\section{Production of organic fertilizer by aerothermal composting of pruning waste}

\section{Diana Mayery Albarracín Sánchez ${ }^{1 *}$, Alba Lucía Roa Parra ${ }^{2}$, Fredy Solano Ortega ${ }^{3,}$ Gladys Montañez Acevedo4}

\begin{abstract}
${ }^{1}$ Maestría en Biología Molecular y Biotecnología, Universidad de Pamplona. Grupo de Investigación en Recursos Naturales. dianamayery@gmail.

${ }^{2}$ Directora Grupo de Investigación en Recursos Naturales, Universidad de Pamplona. albalurp19@gmail.com

${ }^{3}$ Coordinador de Laboratorios, Universidad de Pamplona. Grupo de investigación en Recursos Naturales. fredysolanoortega@gmail.com

${ }^{4}$ Docente Departamento de Matemáticas, Universidad de Pamplona. Grupo de Investigación en Recursos Naturales. gmontaneza@gmail.com
\end{abstract}

\section{Resumen}

Los recursos naturales son la base de la economía, su uso genera residuos que no siempre son manejados adecuadamente. En la Universidad de Pamplona se produce alrededor de 1.000 Kilos / mes de residuos sólidos orgánicos generando un alto impacto en el medioambiente. El propósito de la investigación fue producir abono por compostaje aerotérmico de residuos de poda. Inicialmente el material recolectado fue triturado a un tamaño entre $2-5 \mathrm{~cm}$ utilizando un molino desintegrador forrajero. Posteriormente se elaboraron tres pilas de $1,50 \mathrm{~m}$ de alto y $1,50 \mathrm{~m}$ de diámetro, proporción de $\mathrm{C}: \mathrm{N}$ de $35: 1$ y se ajustó la humedad a $70 \%$. Se realizaron manualmente volteos cada semana durante tres meses y mediciones diarias de temperatura, $\mathrm{pH}$ y humedad. Se tomaron semanalmente muestras para análisis microbiológicos y fisicoquímicos. La calidad del abono producido se evaluó bajo la normatividad existente, NTC 5167. Para observar la variación por pila y en el tiempo, de las variables mencionadas, se aplicó la prueba de Friedman, $(\alpha=5 \%)$ no mostrando diferencias significativas en las variables dependientes por pila, mientras que por el factor tiempo si se presentaron. El Coeficiente de Spearman (rho) mostró que existe una correlación significativa entre algunos grupos microbianos analizados, como por ejemplo Bacterias Coliformes Totales (BCT), Bacterias Coliformes Totales (BCF) y Bacterias Amilolíticas (BA) y
Bacterias Coliformes Totales Bacterias Celulolíticas (BC), entre otras e igualmente se hizo para las variables físico-químicas mostrando correlaciones significativas entre algunas, como: $\mathrm{pH}$ y $\mathrm{P}$, Conductividad y Humedad. El abono producido mostró el cumplimiento de los parámetros microbiológicos exigidos demostrando la eficiencia del proceso aerotérmico.

Palabras clave: residuos sólidos orgánicos, microorganismos, nutrientes, tratamiento de residuos

\begin{abstract}
Natural resources are the basis of the economy, their use generates waste that is not always handled properly. At the University of Pamplona, around 1,000 kilos of organic solid waste are produced that generate a strong impact on the environment. The purpose of the research was directed to the production of fertilizer by aerothermic composition of pruning waste. Initially the collected material was crushed to a size between 2 - 5 $\mathrm{cm}$ using a forage disintegrator mill. Subsequently, three batteries of $1,50 \mathrm{~m}$ high and $1,50 \mathrm{~m}$ in diameter were prepared, with a $\mathrm{C}: \mathrm{N}$ ratio of $35: 1$ and the humidity was adjusted to $70 \%$. Manually volts were made every week for three months and daily measurements of temperature, $\mathrm{pH}$ and humidity. Samples were taken into consideration for microbiological and physicochemical analyzes. The
\end{abstract}


quality of the produced fertilizer was evaluated under the existing regulations, NTC 5167. To observe the variation by pile and in time, of the mentioned variables, applied the Friedman test, $(\alpha=5 \%)$ there were no significant differences in the variables dependent by stack, while by the time factor if it occurs. The Spearman Coefficient showed that there is a significant correlation between some microbial groups analyzed; It was also done for the physicalautomatic variables showing correlations among others, such as: $\mathrm{pH}$ and $\mathrm{P}$, Conductivity and Humidity. The fertilizer produced showed compliance with the microbiological parameters required demonstrating the efficiency of the aerothermal process.

Keywords: fertilizer, microorganisms, nutrients, waste treatment

\section{Introducción}

La urbanización, industrialización y modificación del consumo de las sociedades han sobreexplotado los recursos naturales y el aumento de la producción de residuos, especialmente aquellos que no se reutilizan o se eliminan naturalmente (Patiño, 2008). Esto ha representado un problema debido a los altos volúmenes de residuos sólidos generados por los ciudadanos pues cuando el manejo de éstos no es el adecuado, puede ocasionar problemas sanitarios que afectan la salud de los pobladores y al medio ambiente. Aquellos residuos provenientes de actividades domésticas, agrícolas y forestales están dentro del $46 \%$ de los residuos sólidos producidos por lo que algunos países del mundo planean actividades y normas para la disposición de los residuos que conllevan a multas o recompensas a los pobladores con el objetivo de reducir, reutilizar y reciclar los residuos (Hoornweg \& Bhada-Tata, 2012). El incentivo de prácticas de preservación de los recursos naturales y de uso adecuado y eficiente de los residuos es fundamental pues se considera que estos son una fuente potencial de nutrientes para las plantas a través de su reciclaje por compostaje (Kowalchuk et al., 1999) El compostaje aerotérmico es la descomposición biológica aerobia controlada en donde parte de la materia orgánica es transformada a sustancias estables parecidas a los ácidos húmicos (Farrell \& Jones, 2009) donde se permite el desarrollo de temperaturas óptimas para las bacterias termófilas (Saveyn \& Eder, 2014); adicionalmente son una herramienta biotecnológica en
157

la transformación de los residuos sólidos orgánicos en productos agrícolas apropiados (Baffi et al., 2007). En cuanto a los usos potenciales, el compost es un acondicionador orgánico natural del suelo, pues mejora a mediano y largo plazo las propiedades físicas, químicas y biológicas además aumenta la porosidad, disminuye la densidad aparente, consolida la estructura y consistencia, incrementa la capacidad de intercambio catiónico, la concentración de algunos nutrientes esenciales y su actividad biológica (Bohórquez, Puentes, \& Menjivar, 2014; Quiroz \& Perez, 2013) En Colombia esta práctica ha sido más usada en los últimos años a tal punto que en diferentes regiones existen plantas de tratamiento de residuos especialmente agrícolas cuya materia prima depende de las cadenas productivas de cada región. Los productos del compostaje se utilizan principalmente en los propios cultivos de quienes los procesan. En la actualidad el mercado empieza a expandirse a otros cultivadores que no pueden producir su propio biocompostaje, además de conformarse la Asociación Nacional de Compostajes, cuya meta es exportar este producto de acuerdo a los estándares internacionales (Libreros \& Salamanca, 2012). Con el objetivo de fomentar el proceso en la Universidad de Pamplona se realizó el presente estudio donde se determinó el comportamiento de las características físico-químicas y microbiológicas durante la elaboración del bioabono por compostaje aerotérmico a partir de residuos de poda del campus universitario y se realizó una evaluación de acuerdo a la normatividad colombiana vigente.

\section{Metodología}

\subsection{Descripción del sitio}

El trabajo se realizó en las instalaciones de la Universidad de Pamplona, Pamplona, Norte de Santander Colombia, ubicada en las coordenadas $7^{\circ}$ 23'13. 69' ' latitud N; 72॰ 38' 55. 61', de longitud O, con una altura de $2.380 \mathrm{msnm}$ y una temperatura promedio de $16^{\circ} \mathrm{C}$.

\subsection{Obtención del bioabono}

Para la obtención de bioabono, se aplicó la biotecnología del compostaje aerotérmico. Inicialmente se recolectó material de poda del campus central y se 
158

trasladó al vivero donde se secó naturalmente. Las unidades experimentales consistieron en tres pilas uniformes en composición y dimensiones: Relación carbono nitrógeno 35 , tamaño de partícula $2-5 \mathrm{~cm}$, altura y diámetro de la pila: $1,5 \mathrm{~m}$, humedad $70 \%$ y temperatura ambiental $19+/-2^{\circ} \mathrm{C}$. Las pilas fueron ventiladas a través de volteos manuales semanales durante tres meses para facilitar la aireación y evitar la formación de núcleos de anaerobiosis. Durante el bioproceso se realizaron mediciones diarias de temperatura, $\mathrm{pH}$ y humedad. Semanalmente fueron tomadas muestras para análisis físico-químico: conductividad (potenciómétrico), carbono orgánico (método Walkley-Black modificado por calorimetría), nitrógeno (método de Kjeldahl), fósforo (colorimétrico), capacidad de intercambio catiónico (Método IGAC), calcio, magnesio, potasio, cadmio, hierro, zinc, cobre y manganeso (Espectrofotometría de absorción atómica y colorimetría) (Jackson, 1982); y microbiológico (bacterias coliformes totales y fecales, aerobios mesófilos, termófilos, mohos y levaduras, celulolíticos, amilolíticos, amonificantes y nitrificantes) (Recuento en placa) (Corry, Jarvis, Passmore, \& Hedges, 2007).

El abono producido se evaluó como producto orgánico usado como abono o fertilizante comparando los análisis físico-químicos y microbiológicos obtenidos con la Norma NTC 5167 de 2011.

\section{Análisis estadístico}

En esta investigación se evaluó el resultado del abono obtenido mediante el proceso de compostaje aerotérmico (CA) basado en las características físicoquímicas y microbiológicas de la normatividad vigente (ICONTEC. NTC 5167 de 2011). Para el análisis de los resultados se aplicó, la prueba no paramétrica de Friedman, con el fin de observar el comportamiento de las variables físico-químicas y microbiológicas; adicional se calculó el Coeficiente de correlación de Spearman durante un periodo de tres meses en las tres pilas. El nivel de significancia fue del 5\%. El programa estadístico utilizado para el procesamiento fue el SPSS.

\section{Resultados y Discusión}

Los residuos de poda poseen microbiota nativa, la cual en las primeras etapas del bioproceso se multiplica cambiando las condiciones del medio mediante la realización de procesos metabólicos exotérmicos, los cuales aumentan la temperatura y favorece el crecimiento de los termófilos. La Figura 1 muestra los resultados obtenidos, en la cual la temperatura de las tres pilas, ascendio progresivamente, entre los días $7 \mathrm{y}$ 11 alcanzando valores de $70^{\circ} \mathrm{C}$, condición óptima para eliminar patógenos parásitos y malezas (Pierre et al., 2009). Luego disminuyo a $40^{\circ} \mathrm{C}$ en el día 15 y se mantuvo estable a partir del día 45 en $20^{\circ} \mathrm{C}$. Estos eventos se ven favorecidos por la humedad del sistema, la cual se mantuvo alrededor del $70 \%$.

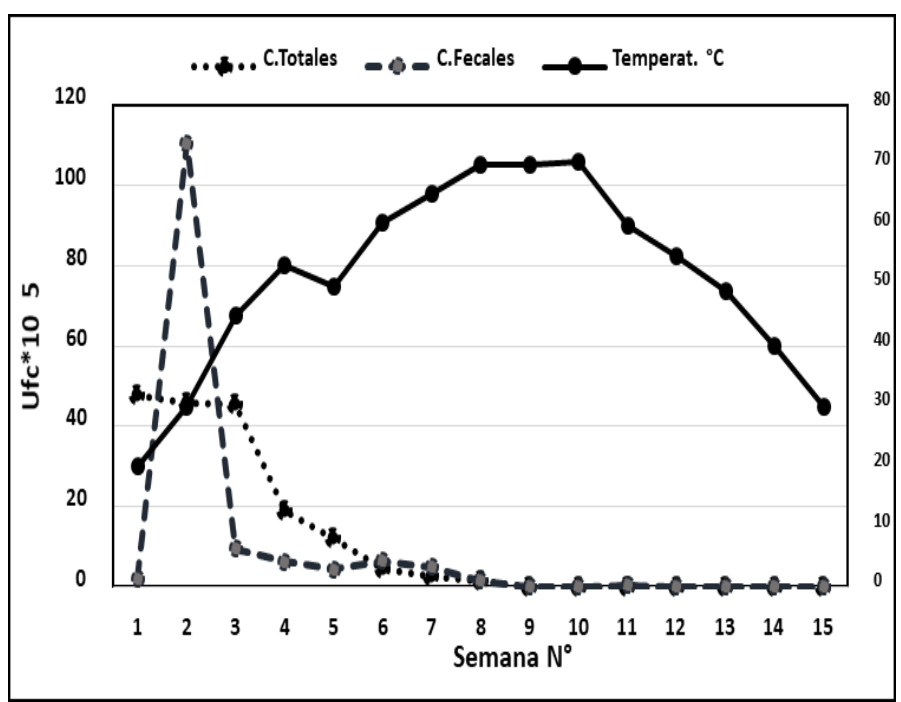

Figura 1. Comportamiento de las Bacterias coliformes totales y Fecales en función de la temperatura

El carbono orgánico mostró una disminución en el tiempo sugiriendo procesos de mineralización con la consecuente pérdida de $\mathrm{C}$ por volatización (SantamaríaRomero, Ferrera-Cerrato, Almaraz-Suárez, GalvisSpinola, \& Berois-Boullard, 2001). Aunque el porcentaje de Nitrógeno alcanzado $1.04 \%$ responde a la normatividad (NTC 5167), la relación C:N se mantuvo sobre 30, lo que puede generar problemas de disponibilidad de nitrógeno del suelo; así los microorganismos al tener abundante alimento incrementan el consumo energético del suelo para su desarrollo y provocan deficiencias para las plantas; se sugiere ajustar valores de $\mathrm{C}: \mathrm{N}$ menores al inicio del bioproceso para alcanzar valores menores de 12 al final como indicador de madurez alcanzable (Flavel \& Murphy, 2006). La conductividad eléctrica como medida indirecta de la cantidad de sales en el suelo mostró valores entre 1.500 y 2000 milimhos/cm lo que puede provocar problemas para el desarrollo de 
159

cultivos. La capacidad de intercambio catiónico (CIC) relacionada con la capacidad de retener los elementos necesarios para nutrir las plantas registró valores entre 6.000 y $10.000 \mathrm{meq} / 100 \mathrm{ml}$, favoreciendo la fertilidad del suelo. El P presentó un valor de $0,013 \%$, valor inferior a lo reportado por (Hernández-rodríguez, Hernández-tecorral, Rivera-figueroa, María, \& Dámaris, 2013). En suelos básicos, como en el caso estudio, puede producirse una fijación lenta e irreversible de una parte del $\mathrm{P}_{2} \mathrm{O}_{5}$, en forma de fosfatos tricálcicos no recuperables. Se reportó un valor de 0,43 \% para $\mathrm{K}$ inferior a lo reportado por (Castillo, Hernández, Dominguez, \& Ojeda, 2010; Hernándezrodríguez et al., 2013). Los valores alcanzados por los micronutrientes son los siguientes: El Ca 10,75\%, valor superior a lo reportado por Ducasal, (2002); el Mg 4,23 , valor superior a lo reportado por Fricke \& Vogtmann (1993); los valores de Mn 16\%, Zn 171\%, $\mathrm{Fe} 4,51 \%$ y $\mathrm{Cu} 0,094 \%$ presentaron un valor significativamente inferior a lo registrado por (Hernández-rodríguez et al., 2013).

Con relación al comportamiento de las poblaciones microbianas durante el proceso de compostaje se observó: Las bacterias mesófilas (BCT y BCF) inicialmente presentaron un incremento por la utilización de los azucares simples (semana 2, 3200000 UFC/g) (De Carlo et al, 2015); agotados los nutrientes dieron paso a microorganismos que utilizaron otras fuentes carbonatadas, las amilolíticas (semana 7 y 8 , $480 \mathrm{UFC} / \mathrm{g}$ ); posteriormente proliferaron las celulolíticas que degradan materiales más complejos (semana 12, 27000 UFC/g) (Frioni Lillian, 1999). Los cambios de escenarios nutricionales junto a las reacciones bioquímicas exotérmicas promovieron la proliferación de las bacterias termófilas (semana 10, $60000 \mathrm{UFC} / \mathrm{g}$ ) y sanearon la pila (Moreno, López, Vargas-García, \& Suárez-Estrella, 2013); esto se evidencia por la disminución de las bacterias coliformes totales y fecales (semana 14 y 15 , menos de $3 \mathrm{UFC} / \mathrm{g}$ ) y las bacterias mesófilas, las cuales registraron los valores más bajos (semana 10, 16000 UFC/g) Figura 1. El comportamiento de los mohos y levaduras permitió observar que no desaparecieron durante el proceso, pero si disminuyó la población durante la fase termofílica. La población inicial fue elevada, probablemente debido a la presencia en la materia prima de residuos vegetales con $\mathrm{pH}$ bajo, lo que favoreció el crecimiento de levaduras (Choi \& Park, 1998; Ryckeboer, Mergaert, Coosemans, Deprins, \& Swings, 2003). A medida que el $\mathrm{pH}$ aumentaba con el tiempo durante el compostaje, se ejerció una presión negativa sobre las levaduras. Los microrganismos capaces de transformar nitrógeno orgánico en el amonio (o amoníaco) presentaron la máxima expresión de crecimiento durante la semana 9 (208000 UFC/gr) (Geisseler, Horwath, Joergensen, \& Ludwig, 2010).

La interdependencia entre variables descrita se evidenció con el Coeficiente de Spearman, el cual mostró la existencia de correlación significativa y directamente proporcional entre algunos grupos microbianos analizados, como por ejemplo BCT y BCF (rho:0.943); BCT y BA (rho: 0.943); ВCT y BC (rho:0.8); BCT y Mohos y Levaduras (rho: 0.647); y correlación significativa inversamente proporcional entre Bacterias termófilas y Mohos y Levaduras (rho: $0.814)$ entre otras.

La Tabla 1 muestra los parámetros de la Norma NTC 5167 Productos para la industria agrícola, Productos orgánicos usados como abono $\mathrm{o}$ fertilizantes y enmiendas $\mathrm{o}$ acondicionadores del suelo y los resultados del bioabono producido. Los valores obtenidos cumplen ampliamente con la norma, denotando que el compostaje aerotérmico se constituye en una estrategia biotecnológica para ser aplicada en la biotransformación de los residuos sólidos de poda, y cuyo producto puede ser reutilizado en el mantenimiento de los jardines de la Universidad de Pamplona.

\begin{tabular}{|l|l|l|}
\hline Parámetro & $\begin{array}{l}\text { Bioabono } \\
\text { producido }\end{array}$ & NTC 5167 \\
\hline $\begin{array}{l}\text { Nitrógeno. N total } \\
(\%)\end{array}$ & 1,04 & Menor 1.0 \\
\hline Fósforo. $\mathrm{P}_{2} \mathrm{O}_{5}(\%)$ & 0,013 & Menor 1.0 \\
\hline Potasio. $\mathrm{K}_{2} \mathrm{O}(\%)$ & 0,43 & Menor 1.0 \\
\hline $\mathrm{CIC}(\mathrm{meq} / 100 \mathrm{~g})$ & 8,5 & Mínimo 30 \\
\hline Humedad $(\%)$ & 30 & $20-35$ \\
\hline pH $\left({ }^{\circ} \mathrm{C}\right)$ & 8,6 & Mayor de 5 \\
\hline Salmonella $\mathrm{sp}$ & $\begin{array}{l}\text { Ausente en } \\
25 \mathrm{~g}\end{array}$ & Ausente en 25 g \\
\hline Coliformes totales & $10 \mathrm{UFC} / \mathrm{g}$ & $\begin{array}{l}\text { Menos de } 1000 \\
\text { UFC/g }\end{array}$ \\
\hline $\begin{array}{l}\text { Huevos de } \\
\text { helmintos viables }\end{array}$ & 0 & $\begin{array}{l}\text { Menos de } 1 \text { en } 4 \mathrm{~g} \\
\text { de material seca }\end{array}$ \\
\hline Fitopatógenos & Ausente & Ausente \\
\hline
\end{tabular}

Tabla 1. Resultados del bioabono producido comparado con la Norma NTC 5167

\section{Conclusiones}


160

Durante el proceso de compostaje aerotérmico se desarrollaron las fases mesofílica, termofílica y de enfriamiento permitiendo la sanidad de la pila.

Los residuos sólidos orgánicos de poda producidos en la Universidad de Pamplona se constituyen en una importante materia prima para la producción de bioabono debido a la riqueza en nutrientes y podrá ser utilizado en las labores propias de ornamentación y reforestación en el marco de una universidad sostenible y sustentable.

\section{Referencias Bibliográficas}

Baffi, C., Dell\&apos;Abate, M. T., Nassisi, A., Silva, S., Benedetti, A., Genevini, P. L., \& Adani, F. (2007). Determination of biological stability in compost: A comparison of methodologies. Soil Biology and Biochemistry, 39(6), 1284-1293. https://doi.org/10.1016/j.soilbio.2006.12.004.

Bohórquez, A., Puentes, Y. J., \& Menjivar, J. C. (2014). Evaluación de la calidad del compost producido a partir de subproductos agroindustriales de caña de azúcar Quality evaluation of compost produced from agro-industrial byproducts of sugar cane. Corpoica Ciencia y Tecnología Agropecuaria, 15(1), 73-81.

Castillo, H., Hernández, A., Dominguez, D., \& Ojeda, D. (2010). Effect of Californian Red Worm (Eisenia foetida) on the Nutrient Dynamics of a Mixture of Semicomposted Materials. Chilean Journal of Agricultural Research, 70(3), 465-473. https://doi.org/10.4067/S0718-

58392010000300014.

Choi, M. H., \& Park, Y. H. (1998). The influence of yeast on thermophilic composting of food waste. Letters in Applied Microbiology, 26(3), 175-178. https://doi.org/10.1046/j.1472-765X. 1998. 00307 .x.

Corry, J. E. L., Jarvis, B., Passmore, S., \& Hedges, A. (2007). A critical review of measurement uncertainty in the enumeration of food microorganisms. Food Microbiology, 24(3), 230-253. https://doi.org/10.1016/j.fm.2006.05.003.
Ducasal, R. R. (2002). Biofertilizantes. Ganadería Integral Vizur, 1.

De Carlo, E. B., Rosa, A. T., Benintende, S., Cariello, M. E., Castañeda, L., Figoni, E., ... \& Mascheroni, F. (2015). Estudio de la publación microbiana en las etapas iniciales del compostaje. Ceres, 48(280).

Farrell, M., \& Jones, D. L. (2009). Critical evaluation of municipal solid waste composting and potential compost markets. Bioresource Technology, 100(19), 4301-4310. https://doi.org/10.1016/j.biortech.2009.04.029.

Flavel, T. C., \& Murphy, D. V. (2006). Carbon and Nitrogen Mineralization Rates after Application of Organic Amendments to Soil. Journal of Environment Quality, 35(1), 183. https://doi.org/10.2134/jeq2005.0022.

Frioni Lillian. (1999). Procesos Microbianos. Procesos Microbianos.

Fricke, K. and H. Vogtmann. 1993. Quality of source separated compost. BioCycle 34: 64.

Geisseler, D., Horwath, W. R., Joergensen, R. G., \& Ludwig, B. (2010). Pathways of nitrogen utilization by soil microorganisms - A review. Soil Biology and Biochemistry, 42(12), 2058-2067. https://doi.org/10.1016/j.soilbio.2010.08.0 21.

Hernández Garcia Jessika Andrea ., Ariza Garcia Jherson, Cano Calle Herminsul de Jesús ,Contreras Pineda Jorge .2013.Estandarización de una técnica para la certificación de jardines clonales de plantas de cacao(Theobroma cacao ) usando marcadores moleculares rapd. Bistua:Revista de la Facultad de Ciencias Básicas.10(2):75-84

Hernández-Rodríguez, Ofelia Adriana, HernándezTecorral, Ana, Rivera-Figueroa, César, Arras-Vota, Ana María, \& Ojeda-Barrios, Dámaris. (2013). Calidad nutrimental de cuatro abonos orgánicos producidos a partir de residuos vegetales y pecuarios. Terra Latinoamericana, 31(1), 35-46. Recuperado en 12 de junio de 2018, de http://www.scielo.org.mx/scielo.php?script=sci_art 
text\&pid=S0187-

$57792013000100035 \& \operatorname{lng}=$ es\&tlng=es.

Hoornweg, D., \& Bhada-Tata, P. (2012). What a Waste: A Global Review of Solid Waste Management. Urban Development Series; Knowledge Papers No.15, World Bank, 116. https://doi.org/10.1111/febs.13058.

Jakcson, M. (1964). Análisis químico de suelos. Barcelona, España. Omega.

Kowalchuk, G. A., Naoumenko, Z. S., Derikx, P. J. L., Felske, A., Stephen, J. R., \& Arkhipchenko, I. A. (1999). Molecular analysis of ammonia-oxidizing bacteria of the beta subdivision of the class Proteobacteria in compost and composted materials. Applied and Environmental Microbiology, 65(2), 396-403.

Libreros, S., \& Salamanca, S. (2012). Compostaje de residuos industriales en Colombia. Revista Tecnicaña, 28, 15-20.

Moreno, J., López, M. J., Vargas-García, M. C., \& Suárez-Estrella, F. (2013). Recent advances in microbial aspects of compost production and use. Acta Horticulturae, 1013, 443-458.

Patiño, A. C. V. (2008). La gestión de los residuos sólidos urbanos en la ciudad de Hannover: un modelo exitoso/The urban waste manegement in Hannover City: a successful model. In Anales de Geografía de la Universidad Complutense (Vol. 28, No. 1, p. 163). Universidad Complutense de Madrid.

Pierre, F., Rosell, M., Quiroz, A., \& Granda, Y. (2009). Evaluación química y biológica de compost de pulpa del café en caspito municipio Andrés Eloy Blanco, estado Lara, Venezuela. Bioagro, 21(2), 105-110.

\section{1}

Quiroz, I., \& Perez, A. (2013). Vinaza y compost de cachaza: efecto en la calidad del suelo cultivado con caña de azúcar. Revista Mexicana de Ciencias Agricolas, 5, 1069-1075.

Ryckeboer, J., Mergaert, J., Coosemans, J., Deprins, K., \& Swings, J. (2003). Microbiological aspects of
161

biowaste during composting in a monitored compost bin. Journal of Applied Microbiology, 94(1), 127-137. https://doi.org/10.1046/j.13652672.2003.01800.x.

Santamaría-Romero, S., Ferrera-Cerrato, R., AlmarazSuárez, J. J., Galvis-Spinola, A., \& BeroisBoullard, I. (2001). Dynamics and relationship among microorganisms, C-Organic and N-Total during composting and vermicomposting. Agrociencia, 35(4), 337-384. Retrieved from http://www.redalyc.org/pdf/302/30235401.pdF.

Saveyn, H., \& Eder, P. (2014). End-of-waste criteria for biodegradable waste subjected to biological treatment (compost \& digestate): Technical proposals. Publications Office of the European Union, Luxembourg Google Scholar. https://doi.org/10.2791/6295

Técnica Colombiana, N. N. 5167. (2011). Productos para la industria agrícola. Productos orgánicos usados como abonos o fertilizantes y enmiendas o acondicionadores de suelo. Bogotá: Instituto Colombiano de Normas Técnicas y CertificaciónICONTEC.

Albarracín Sánchez D. M. Microbiologa con Énfasis en Alimentos.Universidad de Pamplona. Estudiante Maestría en Biología Molecular y Biotecnología, Universidad de Pamplona. Grupo de Investigación en Recursos Naturales.dianamayery@gmail.

Roa Parra A.L. Bacterióloga y Laboratorista Clínico UIS.Magister en Microbiología Universidad Javeriana-UIS. Especialista en Administración y Docencia Universitaria. Universidad de Santander. Doctora en Educación UPEL.Directora Grupo de Investigación en Recursos Naturales Universidad de Pamplona. Coordinadora línea de Investigación Biotecnología. Profesora Titular de la Universidad de Pamplona. Facultad de Salud. Departamento de Bacteriología y laboratorio Clínico. Consultar: http://scienti.colciencias.gov.co:8081/cvlac/visuali zador/generarCurriculoCv.do?cod_rh=0000259217

Solano, Ortega F. Licenciado en Biología - Química . Universidad de Pamplona. Especialista en 
162

Educación y Gestión Ambiental (1998), Universidad de Pamplona. Coordinador de Laboratorios, Universidad de Pamplona. Profesor Titular de la Universidad de Pamplona. Facultad de Ciencia Básicas. Departamento de Química. Grupo de investigación en Recursos Naturales. fredysolanoortega@gmail.com

Montañez, Acevedo G. Licenciada en Matemáticas y Estadistica. Universidad Pedagógica y Tecnológica de Colombia. Especialista en Estadística. Universidad Nacional de Colombia. Magister en Matemáticas. Universidad Nacional Experimental del Táchira. Master en Análisis de datos. Universidad Nacional España a Distancia. Docente Departamento de Matemáticas, Universidad de Pamplona. Grupo de Investigación en Recursos Naturales. gmontaneza@gmail.com

*Para citar este artículo: Albarracín Sánchez D.M.; Roa Parra A.L.; Solano Ortega F.; Montañez Acevedo G.Production of organic fertilizer by aerothermal composting of pruning waste.. Revista Bistua.2018.16(1):156-162.

+ Autor para el envió de correspondencia y la solicitud de las separatas: Albarracín Sánchez D.M. Maestría en Biología Molecular y Biotecnología, Universidad de Pamplona. Grupo de Investigación en Recursos Naturales. dianamayery@gmail.

Recibido: Enero 18 de 2017

Aceptado:Febrero 11 de 2018 